\title{
Cancer Causing Chemicals
}

\author{
Maher Soliman
}

Additional information is available at the end of the chapter

http://dx.doi.org/10.5772/intechopen.71560

\begin{abstract}
Carcinogens are substances that induce cancer by damaging the genome or through disruption of cellular metabolic processes. Some compounds interact directly with DNA, while others are activated to reactive molecules that can bind with DNA by covalent adducts causing mutations in genes crucial to biological processes. Cigarette smoke is by far the most important and notorious carcinogen. Cigarette smoke contains many carcinogenic substances including polycyclic aromatic hydrocarbons (PAHs), which are known to cause cancer. Nitrites, which are present in many foods, are converted into nitrous acid in the stomach and may then react with amines in food to produce nitrosamines, which are carcinogenic. Aflatoxins produced by Aspergillus flavus are promoter for hepatic cancer. Several cytotoxic drugs are carcinogens especially alkylating agents that interact with DNA. Individuals exposed to certain pesticides may be at risk to the development of certain cancers. Inorganic arsenic exposure has been suggested to be associated with the development of several cancers. Sufficient evidence indicated an association between dioxins and various cancers including soft tissue sarcoma, lymphoma and leukemia. Asbestos has been found to be significantly associated with lung cancer and mesothelioma.
\end{abstract}

Keywords: aflatoxins, cancer, carcinogens, carcinogenesis, chemicals, smoking

\section{Introduction}

Worldwide, cancer is a leading cause of mortality [1]. According to GLOBOCAN estimates, about 14.1 million new cancer cases and 8.2 million deaths occurred in 2012 worldwide [1]. The incidence and mortality rates of cancer differ between countries. However, over the last decades, the burden of cancer has shifted to less developed countries due to later stage of diagnosis and unavailability of treatment. Currently about $57 \%$ of cancer cases and $65 \%$ of cancer deaths occurs in less developed countries [1,2]. The cancer profile also varies between countries. The incidence rates of prostate, colorectal, female breast and lung cancer are several 
times higher in more developed countries compared with less developed countries. Liver, stomach, and cervical cancers are more frequent in less developed countries; these cancers are predominantly attributable to infection. However, lung cancer remains the leading cause of cancer death globally [1].

The burden of cancer is rising because of the aging of the population, and increasing exposure to established carcinogenic chemicals, viruses and radiations, as well as adoption of unhealthy lifestyle behaviors such as smoking, alcohol intake, overweight, and limited physical activity. Moreover, the success of screening programs and earlier detection of cancer have contributed to the rise of cancer problem $[1,2]$.

Carcinogens are substances which induce cancer, by damaging the genome or through disruption of cellular metabolic processes. Carcinogens have usually an insidious toxic effect rather than an acute toxic effect. Carcinogens could be either from synthetic chemicals or natural substances. Carcinogens can be classified as genotoxic or nongenotoxic agents according to mechanism of carcinogenesis. Genotoxins bind directly to DNA causing irreversible damage to the genome like polycyclic aromatic hydrocarbons (PAHs). Nongenotoxins do not directly affect DNA but could promote growth, like hormones and some organic compounds. Procarcinogens are not carcinogenic themselves, but turn into carcinogenic substances in the body, e.g. nitrates taken in the diet change into nitrosamines which is carcinogenic. Co-carcinogens are substances that promote the activity of other carcinogens in causing cancer but they are not carcinogenic on their own [3].

Chemical carcinogenesis was first described in 1775 by an eminent English physician and surgeon, Percivall Pott who observed the occurrence of cancer of the scrotum in a number of his patients who were chimney sweepers when they were young $[3,4]$. Accordingly, Pott suggested that the occupation of these men as young boys and their exposure to large amounts of soot could be the causative agent of the cancer. One hundred years later, the high incidence of skin cancer among certain German workers was recognized to be associated to their exposure to coal tar, the chief constituent of the chimney sweeps' soot [5].

Only after 140 years of Dr. Pott's report of the association of epidermal cancer of the scrotum with the exposure to soot from the combustion of coal, the first experimental laboratory animal study on carcinogenesis was reported. In 1915, Yamagawa and Ichikawa first published a comprehensive paper describing the production of a malignant epidermal neoplasm by repeatedly applying crude coal tar to the ears of rabbits for a number of months [6]. Afterwards, several studies tried to define and isolate the causative carcinogenic substance from the crude tar. In 1930s, the first carcinogenic chemical compound, dibenz[a,h]anthracene was produced, followed by several polycyclic aromatic hydrocarbons (PAHs) were isolated from active crude tar fractions.

Since 1971, The International Agency for Research on Cancer (IARC) has evaluated more than 1000 agents, and has classified them into five groups as follows [7]:

- Group 1: Carcinogenic to humans (120 agents).

- Group 2A: Probably carcinogenic to humans (81 agents) 
- Group 2B: Possibly carcinogenic to humans (299 agents)

- Group 3: Not classifiable as to its carcinogenicity to humans (502 agents)

- Group 4: Probably not carcinogenic to humans (1 agent)

\section{Mechanisms of action of carcinogens}

Carcinogenesis could be classified into four phases; initiation, promotion, progression and metastasis. Carcinogenic chemicals can initiate and/or promote this process by affecting the expression and activity of certain genes responsible for cell growth, differentiation, DNA repair, cell-cycle control, and apoptosis. Some compounds interact directly with DNA, while others are activated to reactive molecules that can bind with DNA by covalent adducts causing mutations in genes crucial to biological processes. Polycyclic aromatic hydrocarbons (PAHs) are known to cause cancer by forming covalent adducts with DNA, resulting in altered cell growth and repair [3].

Chemical carcinogens can target certain genes termed proto-oncogenes and tumor suppressor genes, which when become mutated, allow cells to grow without control like in breast, colon and lung cancers. Two well studied proto-oncogenes are MYC and RAS, which are responsible for regulation of the cell cycle and apoptosis. MYC function is involved in protein-protein interactions with various cellular factors such as in Burkitt's Lymphoma. RAS function as GTP-binding protein; important in signal transduction cascade such as in Pancreatic, Colorectal, Bladder Breast, Kidney, \& Lung Neoplasms; Leukemia; Melanoma [8, 9]. Mutations in these genes can cause dysregulated cell division. The mutant proteins maintain their normal functions but are no longer under control of orders that regulate these processes. The products of RAS gene are essential components of kinase signaling pathways that regulate cell growth and differentiation. Mutations in RAS can be caused by organochlorine pesticide and exposure to arsenic [3].

One of the most well recognized tumor suppressor genes is p53 also known as TP53 or tumor protein (EC:2.7.1.37) is a gene that codes for a protein that regulates the cell cycle and hence functions as a tumor suppression. Under suboptimal conditions such as DNA damage, tumor suppressor genes produce products that inhibit cell division for growth. Mutations in p53 have been discovered in breast cancer and bronchial cancer exposed to organophosphorus pesticides and PAHs [10].

\section{Some chemical carcinogens}

\subsection{Cigarette smoking}

Cigarette smoking is the most important carcinogen. Cigarettes are the predominant type of tobacco product consumed in the world. Worldwide, more than 1 million cancer deaths are attributed to cigarette smoking annually. Cigarette smoking is the major cause of lung cancer. 
Polycyclic aromatichydrocarbons and the tobacco-specific nitrosamine4-(methylnitrosamino)1-(3-pyridyl)-1-butanone are likely to play major roles lung cancer induction [11].

Cigarette smoking is a major risk factor for cancers of oral cavity, oropharynx hypopharynx and Larynx, and the risk is greatly increased by alcohol consumption. Most cases of esophageal cancer, sinonasal and nasopharyngeal cancer, liver cancer, pancreatic cancer, stomach cancer, cervical squamous cell carcinoma, transitional cell carcinomas of the bladder, ureter, and renal pelvis and myeloid leukemia in adults are linked to cigarette smoking [12].

Laboratory studies clearly demonstrate that inhalation of cigarette smoke and topical application of cigarette smoke condensate (CSC) cause cancer in experimental animals. Studies identified the carcinogenic polycyclic aromatic hydrocarbons (PAHs) in cigarette smoke and showed that CSC has both carcinogenic and cocarcinogenic (tumor-promoting activity) effect. Tumors are not induced by the PAHs alone, using doses equivalent to their concentrations in CSC. However, carcinogenesis appears to depend on the composite interaction of the tumor initiators such as PAHs and tumor promoters [12].

Cigarette smoke contains more than 60 carcinogenic compounds that have been evaluated for carcinogenicity in laboratory animals, and 15 of them are considered as carcinogenic to humans. PAHs are a group of compounds produced from tobacco smoking. PAHs have a direct local carcinogenic effect. Benzo[a]pyrene, one of PAHs, has powerful carcinogenic activity to humans. Heterocyclic compounds are also combustion products and include nitrogen-containing analogues of PAHs such as furan, which is carcinogenic to liver [12].

$\mathrm{N}$-nitrosamines are a large group of potent carcinogens. N-nitrosamines in cigarette smoke can induce lung tumors, tumors of the pancreas, nasal cavity, and liver and esophageal tumors. Aromatic amines such as 2-naphthylamine and 4-aminobiphenyl, which is the first identified carcinogens resulting from dye industry exposures, causes bladder cancer. Aldehydes such as formaldehyde and acetaldehyde are commonly present in our daily life from smoking. Other carcinogens present in cigarette smoke are vinyl chloride, and ethylene oxide [12, 13].

Cigarette smoke also contains oxidants such as nitric oxide and free radicals that are involved in oxidative damage produced by cigarette smoke. Cigarette smoke contains diverse carcinogens. PAH, N-nitrosamines, aromatic amines, 1,3-butadiene, benzene, aldehydes, and ethylene oxide are among the most important carcinogenic compounds present in cigarette smoke [12].

\subsection{Polycyclic aromatic hydrocarbons}

Polycyclic aromatic hydrocarbons (PAHs) are a group of organic compounds composed of two or more fused aromatic (benzene) rings containing only carbon and hydrogen atoms. PAHs containing two and three rings are present in vapor phase in atmosphere as they have low molecular weight. However, PAHs with five rings or more are largely bound to particles and considered the most hazardous to humans. PAHs with intermediate molecular weight (four rings) are allocated between vapor and particulate phases. More than 11 carcinogenic PAHs were detected in the air of industrial countries. The most widely-spread PAHs compound is benzo[a]pyrene which is used as a marker for total exposure to carcinogenic PAHs. 
Polycyclic hydrocarbons differ in their carcinogenicity; some have weak carcinogenic effect like, the compound dibenzo[a,c]anthracene, while others have potent carcinogenicity like 3-methylcholanthrene and 7,12-dimethylbenzo[a]anthracene [14].

PAHs are released into the environment form the combustion of carbon containing materials at high temperature. Indoor air contamination by PAHs occurs from indoor emission sources such as smoking, cooking, domestic heating with fuel stoves and open fireplaces, as well as from intrusion of outdoor air [14].

PAHs emissions from motor vehicle, power generation plants, waste incinerators and open burning are considered the main component of outdoor sources in industrialized countries. In developing countries, cooking and heating with solid fuels such as wood, agricultural residues or coal remains the main contributing source of indoor PAHs air pollution [14].

Individual exposure to PAHs occurs via inhalation of air, consumption of food and water, and dermal contact with soil and dust. Indoor air would be the major source contributing to the PAHs exposure through inhalation, as people spent $80-93 \%$ of their time indoors [14].

PAHs are easily absorbed from the gastrointestinal tract of human, as they are highly lipid soluble. Then they are rapidly distributed in a various tissues with a tendency for localization in fatty tissues. PAHs metabolized via the cytochrome P450s and epoxide hydrolase enzymes [14].

The carcinogenic potential of PAHs has been well established for decades, and evidence to date has resulted in many of these compounds being labeled as reasonably carcinogenic. Lung tumors have been detected in animals exposed to PAHs. In vitro studies showed that c-myc expression, adduct formation, and cell-cycle progression are altered in lung epithelial cells exposed to PAHs [15].

The mechanisms of carcinogenesis of PAHs have been extensively investigated. PAHs might cause DNA adducts. Recent studies indicate that PAHs can alter cell signaling cascades that control cell communication, growth, and immune functions. PAHs have been shown to act through nuclear receptors [15].

\subsection{Nitrosamines}

Nitrosamines are a class of approximately 300 compounds and about $90 \%$ of them have been found to be carcinogenic. For example dimethylnitrosamine causes liver cancer, whereas some of the tobacco specific nitrosamines cause lung cancer. Nitrates and nitrites occur naturally in fruit and vegetables, which are considered as an important part of a healthy diet in most countries. Nitrates and nitrites are often used as food additives in processed meats such as ham, bacon, sausages and hot dogs to prevent toxin production by Clostridium Botulinum (the microorganism responsible for botulism), and preserve meat products recognizable appearance and flavor as well [13].

Nitrosamines are produced by chemical reactions of nitrates or its reduced form nitrites with amines in the meat during its processing, storage, and cooking. N-nitrosodimethylamine 
(NDMA) is one of the most frequently occurring nitrosamines in our dietary foods. NDMA is a potent carcinogen, associated with increased risk of malignant tumors of liver, lung, and stomach [13].

Nitrates $\left(\mathrm{NO}_{3}\right)$ and nitrites $\left(\mathrm{NO}_{2}\right)$ are inorganic compounds, composed of a single nitrogen atom $(\mathrm{N})$ and a number of oxygen atoms $(\mathrm{O})$. It is believed that nitrates themselves are relatively inert, and activated by nitrate reductase enzyme from bacteria in the mouth into to nitrites. Then nitrites are converted to nitrous acid by the acidic juices in stomach, which further reacts with amines to form nitrosamines. The carcinogenesis of nitrosamines could be through gene mutation and DNA adductions. A high consumption of processed meats was correlated to an increased gastric cancer risk, and many people consider nitrates/nitrites as the main reason for that [13].

\subsection{Aflatoxins}

Aflatoxin is a potent human carcinogen. It is a naturally occurring toxic metabolite produced by certain fungi (Aspergillus flavis). Aflatoxins are an interesting example of DNA damaging agents from a natural source. Among the aflatoxins of natural origin, aflatoxin B1 is the most potent hepatocarcinogen and considered to be the most toxic. Aflatoxins are regularly found in improperly stored staple commodities such as cassava, corn, cotton seed, millet, peanuts, rice, sesame seeds, and wheat [16].

Aflatoxins may be metabolized in the liver to a reactive epoxide intermediate or hydroxylated to become the less harmful aflatoxin $\mathrm{M}_{1}$. Aflatoxins are commonly ingested through contaminated food. Animals fed contaminated food can pass aflatoxin transformation products into eggs, milk products, and meat. However the most toxic type of aflatoxin $\mathrm{B}_{1}$, can permeate through the skin. It has been suggested that aflatoxins induce p53 gene mutations in hepatocytes [16].

\subsection{Drugs and chemotherapy}

Many drugs have carcinogenic potential such as intercalating antibiotics or nitroimidazole derivatives like metronidazole. The mechanism of action of nitroimidazoles is through reduction of the nitro group in predominantly anaerobic environments leads to formation of reactive intermediate products and hence destruction of DNA strands. Antimicrobial agents can be directly toxic, can interact with other drugs to increase their toxicity, or can alter microbial flora to cause infection by organisms that are normally saprophytic [17].

The majority of cytostatic agents (like: melphalan, nitrosourea, etoposide) are potentially carcinogenic. Certain tumors have been triggered by chemotherapy. Furthermore, some cytostatic agents have an immunosuppressive effect which renders the organism unable to eliminate mutated cells efficiently.

Owing to increased survival rates after chemotherapy, some patients develop years after primary therapy secondary malignancy. Most of secondary malignancies appear in the first 10 years after chemotherapy, especially after alkylating agents or nitrosourea derivatives. Of the alkylating agents, ALKERAN (melphalan), also known as L-phenylalanine mustard, phenylalanine mustard, LPAM, or L-sarcolysin, is a phenylalanine derivative of nitrogen mustard. 
According to previous studies, alkylating agents such as cyclophosphamide, melphalan or procarbazine have the strongest leukemogenic potential [18].

\subsection{Pesticides}

Pesticides are a group of biologically active natural and synthetic chemicals, which are used to kill unwanted harmful insects, fungi, rodents and plants. All pesticides contain biologically active compounds that are purposely designed to interfere with normal biologic processes in target organisms. Therefore, individuals exposed to certain pesticides may be at risk to the development of certain cancers. Exposure to these pesticides could be through occupational exposures, the ingestion of contaminated food and water, by absorption through the skin, or by inhalation during application [19].

Currently, evidence supports that pesticides containing arsenic and ethylene oxide have the potential to cause cancer in humans. Pesticides may cause cancers by affecting cellular proliferation, apoptosis, and cell communication and inducing oxidative stress through nongenotoxic mechanisms. For example, 1,1,1-Trichloro-2,2-bis( $p$-chlorophenyl)-ethane (DDT) is one of the most well-known organochlorine carcinogenic pesticides. DDT tumorigenesis has been shown to be caused by tissue damage through oxidative mechanisms, alter cell signaling pathways (MAPK) that regulate growth, or activate the oncogene erb-B2 [20].

Though DDT was banned in the United State in the early 1970s, it is still used in other countries, and high levels of DDT has been detected in air, water, soil, plants, animals, and human tissues. The organochlorine pesticide 1,1,1-trichloro-2,2-bis (p-chlorophenyl)-ethane (DDT), is a well-known and widely dispersed "environmental estrogen" (World Health Organization Criteria no. 9; Geneva, Switzerland (1979)".

\subsection{Arsenic}

Inorganic arsenic has been suggested to be a human carcinogen since 1977. Arsenic is emitted into the atmosphere mainly from anthropogenic sources and a small amount from natural sources. Individuals may be exposed to arsenic compounds through contaminated food and drinking water or air emissions from industrial facilities that manufacture pesticides, glass, and cigarette tobacco [21].

Evidence suggests that there is a strong association between arsenic exposure and the development of skin, lung, bladder, kidney, liver, and colon cancers. The mechanism by which arsenic causes cancer is not well understood. It has been proposed that Arsenic does not interact with DNA, but indirectly causes chromosome aberrations, genomic instability, and aberrant DNA methylation in promoter regions of genes [22].

\subsection{Dioxins}

Dioxins are a group of structurally related compounds produced from industrial and combustion activities such as bleaching of paper, the manufacture of some pesticides, waste incineration, and fuels burning. They could be released from natural sources such as volcanic eruptions and forest fires. Dioxins are found in air, soil, water and food sources. Sufficient 
evidence from human epidemiologic and mechanistic studies showed an association between dioxins and various cancers including soft tissue sarcoma, lymphoma, leukemia, and Hodgkin disease. TCDD is the well-studied dioxin, which is known to be human carcinogen [23].

The mechanism responsible for dioxin-mediated carcinogenesis is via activation of the aryl hydrocarbon $(\mathrm{Ah})$ receptor resulting in a wide spectrum of biologic responses, including altered metabolism, growth, and differentiation. It has been suggested that Dioxin alters multiple integrated cell signaling pathways, namely, the MAPK-ERK pathway through activation of tumor necrosis factor-alpha (TNF-a) and epidermal growth factor (EGF). Others suggested mutations in the proto-oncogene H-ras [24].

\subsection{Asbestos}

Asbestos is a group of six naturally occurring fibrous silicate minerals, namely: chrysotile, actinolite, amosite, anthophyllite, crocidolite, and tremolite. Chrysotile constitutes about $90 \%$ of the commercially used asbestos worldwide. Asbestos is released into the environment from natural and man-made sources and has been detected in air, soil, drinking water, food, and medicines. Asbestos toxicity occurs after a long latent period of about 15-40 year after initial fiber exposure. Occupational exposure to any type of asbestos increases the risk of lung cancer and mesothelioma [25].

More than 50 countries have banned the mining and/or use of all types of asbestos. However, past and current occupational asbestos exposures, and non-occupational domestic asbestos exposure originating from existing buildings that contain enormous amounts of the fibers and neighborhood exposures in communities living near asbestos mining, remains a global health challenge [26].

The carcinogenesis of asbestos is not fully understood. However, asbestos is a genotoxic agent that can induce direct DNA damage, gene transcription, and protein expression important in modulating cell proliferation, cell death, and inflammation [25].

\section{Author details}

Maher Soliman

Address all correspondence to: maher.soliman@daad-alumni.de

Oncology Department, Faculty of Medicine, Alexandria University, Alexandria, Egypt

\section{References}

[1] Torre LA, Bray F, Siegel RL, Ferlay J, Lortet-Tieulent J, Jemal A. Global cancer statistics, 2012. CA: A Cancer Journal for Clinicians. 2015;65(2):87-108 
[2] Bray F, Moller B. Predicting the future burden of cancer. Nature Reviews. Cancer. 2006; 6:63-74

[3] Pitot CH, Loeb DD. Fundamentals of Oncology. 4th ed. New York: Marcel Dekker; 2002. 41p

[4] Pott P. The Chirurgical Works of Percivall Pott, Vol. 5, Chirurgical Observations Relative to the Cataract, the Polypus of the Nose, the Cancer of the Scrotum, the Different Kinds of Ruptures, and the Mortification of the Toes and Feet. London: L. Hawes, W. Clarke and R. Collins; 1775

[5] Miller EC. Some current perspectives on chemical carcinogenesis in humans and experimental animals: Presidential address. Cancer Research. 1978;38:1479-1496

[6] Yamagawa K, Ichikawa K. Experimentelle Studie über die Pathogenese der Epithelialgeschwülste. Mitteilungen of Medical Faculty of Imperial University of Tokyo. 1915;15: 295-344

[7] “IARC Monographs”. Monographs.iarc.fr. Retrieved 2017-10-5

[8] Gibbs WW. Untangling the roots of cancer. Scientific American. 2003;289(1):56-65

[9] Kling J. Put the blame on methylation. The Scientist. 2003;16(12):27-28

[10] Barrett JC, Shelby MD. Mechanisms of human carcinogens. Progress in Clinical and Biological Research. 1992;374:415-434

[11] Hecht SS. Tobacco smoke carcinogens and lung cancer. Journal of the National Cancer Institute. 1999;91:1194-1210

[12] Hecht SS. Etiology of cancer: Tobacco. In: TV DV, Lawrence ST, Rosenberg SA, editors. Devita, Hellman \& Rosenberg's Cancer: Principles \& Practice of Oncology. 8th ed. Philadelphia: Lippincott Williams \& Wilkins; 2008. p. 148-165

[13] Oliver CP, Gloria MBA, Barbour JF, Scanlan RA. Nitrate, nitrite and volatile nitrosamines in whey-containing food products. Journal of Agricultural and Food Chemistry. 1995;43:967-969

[14] Air Quality Guidelines for Europe. 2nd ed. Copenhagen: WHO Regional Office for Europe; 2000 Polycyclic Aromatic Hydrocarbons. (WHO Regional Publications, European Series, No. 91)

[15] Menzie CA, Potocki BB, Santodonato J. Exposure to carcinogenic PAHs in the environment. Environmental Science and Technology. 1992;26:1278-1284

[16] McPherson M. Mycotoxin testing with aran gas: Aflatoxin B1 mycotoxin destruction using allotropic oxygen O4, O5, and O6. The Journal of Microbiology. 2015;2(6):00068

[17] Neu HC, Gootz TD. Medical Microbiology. 4th ed. University of Texas Medical Branch; Galveston; 1996. ISBN-10: 0-9631172-1-1 
[18] Jones RB, Frank R, Mass T. Safe handling of chemotherapeutic agents: A report from the Mount Sinai Medical Center. CA: A Cancer Journal for Clinicians. 1983;33:258-263

[19] Alavanja MC, Samanic C, Dosemeci M, et al. Use of agricultural pesticides and prostate cancer risk in the agricultural health study cohort. American Journal of Epidemiology. 2003; 157:800-814

[20] Bounias M. Etiological factors and mechanism involved in relationships between pesticide exposure and cancer. Journal of Environmental Biology. 2003;24:1-8

[21] Guo HR, Wang NS, Hu H, Monson RR. Cell type specificity of lung cancer associated with arsenic ingestion. Cancer Epidemiology, Biomarkers \& Prevention. 2004;13:638-643

[22] Shi H, Shi X, Liu KJ. Oxidative mechanism of arsenic toxicity and carcinogenesis. Molecular and Cellular Biochemistry. 2004;255:67-78

[23] Bertazzi PA, Zocchetti C, Guercilena S, et al. Dioxin exposure and cancer risk: A 15-year mortality study after the "Seveso accident." Epidemiology. 1997;8:646-652

[24] Cole P, Trichopoulos D, Pastides H, Starr T, Mandel JS. Dioxin and cancer: A critical review. Regulatory Toxicology and Pharmacology. 2003;38:378-388

[25] MacDonald L. The health hazards of asbestos removal. JAMA. 1992;267:52-53

[26] Mossman BT, Bignon J, Corn M, Seaton A, Gee JB. Asbestos: Scientific developments and implications for public policy. Science. 1990;247:294-301 